\title{
Assessment of left ventricular myocardial systolic dysfunction in premature ovarian insufficiency using echocardiographic layer-specific myocardial strain imaging
}

\author{
Yu-lin Wang ${ }^{1}$, Lixue Yin ${ }^{1}$, and Mei $\mathrm{Li}^{1}$ \\ ${ }^{1}$ Affiliation not available
}

November 6, 2020

\begin{abstract}
Objective: To explore the myocardial systolic dysfunction in premature ovarian insufficiency (POI) patients with normal left ventricular ejection fraction (LVEF) using echocardiographic layer-specific myocardial strain imaging. Methods: Forty-eight newly diagnosed, untreated POI patients with normal LVEF (POI group) and fifty healthy female volunteers with matching age, height and weight (controls group) were enrolled. Both groups underwent standard transthoracic echocardiography to obtain conventional left ventricular and layer-specific strain parameters, the layer-specific strain parameters including the subendomyocardial global longitudinal strain (GLSendo), the mid-layer myocardial global longitudinal strain (GLSmid), the subepimyocardial global longitudinal strain (GLSepi), the subendomyocardial global circumferential strain (GCSendo), the mid-layer myocardial global circumferential strain (GCSmid), and the subepimyocardial global circumferential strain (GCSepi). Results: The end-diastolic interventricular septal thickness (IVST) in the POI group was significantly thicker than that in the controls group $(\mathrm{P}=0.008)$, and The E, E/A, lateral e' in POI group were significantly lower than those in the controls group $(\mathrm{P}<0.05)$. The GLSendo, GLSmid, GLSepi, GCSendo, GCSmid, and GCSepi in the POI group were significant lower than those in the controls group $\left(\mathrm{P}_{i} 0.05\right)$. Receiver operating characteristic (ROC) curve showed that the area under the curve (AUC) of GLSepi was the largest in diagnosing impaired left ventricular systolic function in POI patients $(\mathrm{AUC}=0.706, \mathrm{P}=0.000)$. Conclusions: POI patients with normal LVEF may suffer with subclinical left ventricular myocardial systolic dysfunction. The echocardiographic layer-specific myocardial strain could be more sensitive to be used to detect the subclinical impairment of left ventricular systolic function in POI patients.
\end{abstract}

\section{INTRODUCTION}

Premature ovarian insufficiency (POI) refers to a clinical syndrome caused by loss of ovarian activity in women before the age of 40 years. POI is mainly manifested as menstrual abnormalities (oligomenorrhea or amenorrhea), increased gonadotrophins such as follicle stimulating hormone (FSH $>25 \mathrm{IU} / \mathrm{L})$, decreased estrogen level, and the population prevalence rate is about $1 \% .{ }^{1}$

The etiology of POI is complex, which can be caused by genetic defects (sex chromosomes and autosomes), immune system diseases, thyroid diseases, environmental factors, and iatrogenic factors. However, there are still some patients with unknown causes. ${ }^{1}$

POI can make adverse effects on women's multiple systems, and the harm to the cardiovascular system will reduce life expectancy. ${ }^{1}$ POI can be used as an independent intermediate risk factor for ischemic heart disease (IHD) and cardiovascular diseases (CVD), ${ }^{2}$ and its pathophysiological mechanism affecting the cardiovascular system is mainly a series of changes caused by the lack of estrogen.

Most women with POI have no obvious precordial discomfort in the early stage, and they often ignore changes in the cardiovascular system. In 2016, the European Society of Human Reproduction and Embryology 
(ESHER) recommended that all women with POI should assess the risk of CVD and receive early hormone replacement therapy (HRT) to control future CVD risk, and POI patients with special types of Turner Syndrome (TS) who have sex chromosomal abnormalities, once diagnosed, should be assessed the CVD risk and CVD-related risk factors annually. ${ }^{1}$

The emerging layer-specific myocardial strain imaging in recent years is based on two-dimensional speckle tracking echocardiography (2D-STE), which can quantify the myocardial strain parameters of each layer, provide more accurate myocardial deformation informations, and provide more guidances for clinical precision treatment. This study intended to use layer-specific myocardial strain to quantitatively assess the subclinical myocardial systolic impairment of the left ventricle in POI women with preserved left ventricular ejection fraction (LVEF-pr), expecting to provide a new non-invasive method for clinical evaluation of early changes in the left ventricular myocardial systolic function in POI women for more precise treatment..

\section{METHODS}

\subsection{Study population}

Forty-eight patients, who were untreated with HRT, and newly diagnosed as POI, from the Menopausal Gynecological Endocrinology Clinic of our hospital were included in the POI group.

All subjects in the POI group underwent sex hormone testing, and patients suspected TS underwent chromosome testing to confirm the diagnosis. And inclusion criteria: Meet the POI diagnostic criteria proposed in the 2016 by ESHER ${ }^{1}$ : oligomenorrhea / amenorrhea at least 4 months, and an elevated FSH $>25 \mathrm{IU} / \mathrm{L}$ on two occasions $>4$ weeks apart. No clinically diagnosed severe cardiovascular diseases, and no obvious clinical manifestations of heart involvement such as chest pain, chest tightness, and palpitations.

Exclusion criteria: Age $>40$ years old, those who have undergone HRT, those who have abnormal menstruation caused by radiotherapy and chemotherapy for malignant tumors. Congenital and acquired heart diseases, hypertension, diabetes, thyroid disease, blood system disease, autoimmune system disease. Patients with abnormal LVEF $(\mathrm{LVEF}<54 \%)^{3}$ or those with poor image quality.

Fifty healthy female volunteers who came to physical examination during the same period and with matched age, height, and weight were selected as the controls group. Inclusion criteria: sinus rhythm, no hypertension, no hyperlipidemia, no diabetes, and normal transthoracic echocardiography. Exclusion criteria: routine transthoracic echocardiography revealed abnormalities or poor image quality.

This study was reviewed and approved by the hospital ethics committee (batch number: No. 265), and all subjects signed an informed consent.

\subsection{Procedures}

All subjects rested peacefully for 10 minutes and then were measured the blood pressure. After that, making them take the left side lying position, breath calmly, be connected to the electrocardiogram, and be performed a complete echocardiography. Using Vivid E9 (GE Healthcare,US), with M5S (probe frequency:1.4-4.6MHz). The M5S probe was used to collect two-dimensional dynamic images of at least three cardiac cycles from the parasternal left ventricle long axis view, apical views, and left ventricular short-axis views. Activate the pulsed wave Doppler (PW) and tissue Doppler imaging (TDI) to collect the mitral valve anterior blood flow spectrum and the velocity of the mitral annulus on the apical 4-chamber view.

EHCO PAC 202 workstation was employed for image postprocessing and measurement of the end-diastolic diameter of left ventricle (LVEDD), end-diastolic thickness of left ventricular posterior wall (LVPWT), and end-diastolic interventricular septal thickness (IVST) at the parasternal left ventricular long axis view. The formulas as below were used to calculate relative wall thickness (RWT) $=2 *$ LVPWT/LVEDD, left ventricular mass $(\mathrm{LVM})=0.8^{*} 1.04 *\left[(\mathrm{LVEDD}+\mathrm{IVST}+\mathrm{LVPWT})^{3}-\mathrm{LVEDD}^{3}\right]+0.6$, body surface area $(\mathrm{BSA})$ $=0.0061 *$ height $(\mathrm{cm})+0.0128^{*}$ weight $(\mathrm{kg})-0.1529$, body mass index $(\mathrm{BMI})=$ weight $(\mathrm{kg}) /$ height $(\mathrm{m})^{2}$, and left ventricular mass index $(\mathrm{LVMI})=\mathrm{LVM} / \mathrm{BSA}$. 
LVEF and left atrial volume (LAV) were measured by the biplane Simpson's method, and left ventricular volume index (LAVI) was calculated by LAV/BSA. ${ }^{3}$

The apical views, left ventricular short-axis views were selected in turn, starting the Q-analysis system of EHCO PAC 202 workstation, entering the 2D-strain to manually trace the boundary of the endocardium and epicardium, determining the region of interest (ROI), and the software would automatically track the movement trajectory of the myocardial spots in each layer, and get the subendomyocardial global longitudinal strain (GLSendo), the mid-layer myocardial global longitudinal strain (GLSmid), the subepimyocardial global longitudinal strain (GLSepi), the subendomyocardial global circumferential strain (GCSendo), the mid-layer myocardial global circumferential strain (GCSmid), and the subepimyocardial global circumferential strain (GCSepi) (Figure 1).

The evaluation of left ventricular diastolic function was based on the evaluation guideline for left ventricular diastolic function proposed by ASE/EACI in 2016. ${ }^{4}$

Using simple random sampling method, 10 cases were randomly selected from POI patients for intraobserver reproducibility test, and another 10 cases were randomly selected for interobserver reproducibility test.

\subsection{Statistical analysis}

SPSS 25.0 was used for statistical analysis in this study,and the datas were expressed as mean +- standard deviation. K-S test was used to determine the distribution type of continuous variables, and Levene test was used to test the homogeneity of variance.

If the datas from two geoups satisfied both normal distribution and homogeneity of variance, a two independent samples t-test was performed; otherwise, the Mann-Whitney U test of two independent samples was used. Receiver operating characteristic (ROC) curve was used to study the diagnostic efficacy of layer-specific strain parameters on left ventricular systolic function impairment in POI patients. Intraclass correlation coefficient (ICC) was used for the reproducibility test.

A $P$-value $<0.05$ was regarded as statistically significant for all analyses.

\section{RESULTS}

\subsection{Routine clinical data}

Among the forty-eight cases in the POI group, the estrogen levels of twenty-two cases were below 37pmol/L (the bottom line of serum sex hormone testing equipment was $37 \mathrm{pmol} / \mathrm{l}$ ), and the estrogen level of other twenty-six cases was $88.37+-48.68 \mathrm{pmol} / \mathrm{l}$, and the FSH level of the POI group was $66.70+-27.89 \mathrm{mIU} / \mathrm{ml}$.

There were five cases of TS patients in the POI group, of which three cases had chromosomes: 45, X, and the other two cases respectively were: 45,X/46,XX/47,XXX; 45,X/46,X,r(X). Among the POI patients, four cases were primary amenorrhea, and the abnormal menstruation history of remaining forty-four cases was 13.07+-9.33 months.

No significant differences in age, BSA, BMI, systolic blood pressure, diastolic blood pressure, and heart rate between the two groups were found (all $P>0.05$ ) (Table 1).

\subsection{Standard echocardiography}

The value of IVST in the POI group was significantly larger than that in the controls group $(P=0.008)$, but no statistical differences between the two groups in LVPWT, LVEDD, RWT, LVM, and LVMI (allP >0.05). The values of E, E/A, lateral e' in POI group were significantly lower than those in controls group (all $P$ $<0.05)$, while no differences in A, septal e', average E/e' and LVEF between the two groups were found $(P$ $>0.05$ ) (Table 2).

According to the left ventricular diastolic function assessment guidelines proposed by ASE/EACI in 2016, ${ }^{4}$ the left ventricular diastolic function in all subjects remained normal, but individual abnormal parameters in 
the POI group (E, E/A, lateral e') still suggested that there were adverse changes in left ventricular diastolic function in POI patients.

\subsection{Layer-spcific myocardial strain}

GLSendo, GLSmid, GLSepi, GCSendo, GCSmid and GCSepi in the POI group were all lower than those in the controls group $(P ; 0.05$ or $P ; 0.001)$, the longitudinal strain and circumferential strain parameters of the two groups all followed the decreasing trend from the subendocardium to the subepicardium (Table 3 ) (Figure 2, Figure 3).

3.4 Efficacy of layer-specific strain parameters in diagnosing the impairment of left ventricular systolic function in POI patients.

ROC analysis showed that the area under the curve (AUC) of GLSepi was the largest in diagnosing impairment of left ventricular systolic function in POI patients (AUC $=0.706, P=0.000$ ), and when the cut-off value was $-20.15 \%$, the sensitivity was $79.9 \%$ and the specificity was $54.7 \%$ (Table 4 ) (Figure 4 ).

\subsection{Reproducibility of layer-specific strains}

Intraobserver and interobserver coefficients of GLSendo, GLSmid, GLSepi, GCSendo, GCSmid, GCSepi were all greater than 0.900 , and the reproducibility of layer-specific strains was acceptable (Table 5).

\section{DISCUSSION}

The concept of premature ovarian failure (POF) appears before POI. POF refers to women with amenorrhea, elevated gonadotropin levels, and decreased estrogen levels before the age of 40, accompanied by various perimenopausal related symptoms (such as hot flashes, insomnia, body pain, etc.), and it is the final stage of POI. ${ }^{5}$ The population prevalence rate of POI is about $1 \%,{ }^{1}$ the incidence of POF before the age of 30 is about 1 before the age of 40 is about $1 \%$, the incidence of POF in patients with secondary amenorrhea is about $4 \%-18 \%$, and the incidence of POF in patients with primary amenorrhea is about $10 \%-28 \%{ }^{6}$

Due to the loss of functional follicles, women with POI are in a state of low estrogen for a long time, causing them to face a series of problems such as infertility and perimenopausal syndrome. In addition, Cardiovascular markers in women with POI have deteriorated, women with POI have a higher risk of IHD and CVD, POI can be used as an independent intermediate risk factor for IHD and CVD. ${ }^{2,7}$

Another study suggested that early menopause was associated with an increased risk of heart failure. ${ }^{8}$ Since most women with POI do not have chest tightness, chest pain and other CVD-related clinical features in the early stage, CVD risk detection is often ignored. LVEF is commonly used in clinical evaluation of left ventricular systolic function, and myocardial strain can more sensitively reflect the subclinical myocardial changes in the early stage of the disease than LVEF. ${ }^{9}$ The layer-specific myocardial strain can more accurately quantitatively analyze the deformation of the endocardium, middle myocardium, and epicardium, and has been more maturely used in the study of various diseases. ${ }^{10-12}$ However, there is still a lack of study reports on layer-specific myocardial strain in POI patients.

In this study, the IVST in the POI group was thicker than that in the controls group $(P=0.008)$, suggesting that women with POI may have left ventricular remodeling. The possible pathophysiological mechanisms are: Estradiol $\left(\mathrm{E}_{2}\right)$ as a fast activator of endothelial nitric oxide synthase (NOs) can stimulate the production of nitric oxide (NO), but women with POI continue to be in a low $\mathrm{E}_{2}$ status, resulting in reduced $\mathrm{NO}$ production by vascular endothelial cells, decreased vascular elasticity and compliance, and resulting in increased cardiac afterload, stimulating myocardial cell hypertrophy, and promoting left ventricular remodeling and myocardial hypertrophy; ${ }^{13-14}$ otherwise low $\mathrm{E}_{2}$ can lead to abnormalities in the renin-angiotensin system (RAS) in the heart and systemic circulation, and enhance the activity of angiotensin converting enzyme (ACE), leading to ventricular remodeling and cardiac hypertrophy. ${ }^{15}$

However, according to adult echocardiographic quantitative measurement guideline proposed by ASE/EACI in 2015 for left ventricular hypertrophy (RWT $>0.42$, female LVMI $>95 \mathrm{~g} / \mathrm{m}^{2}$ ), ${ }^{3}$ All subjects in this study 
did not meet the criteria for left ventricular hypertrophy, but fifteen cases in the POI group (34.25\% of the POI group) reached the criteria for concentric remodeling of the left ventricle (RWT $>0.42$, female LVMI[?] $\left.95 \mathrm{~g} / \mathrm{m}^{2}\right) .^{3}$

In addition, no statistical differences in LVPWT, LVEDD, RWT, LVM, LVMI between the two groups were found $(P>0.05)$, indicating that although POI women had local myocardial thickening, they had not yet caused significant changes in the overall geometry of the left ventricle.

Echocardiography can not only evaluate changes in left ventricular geometry in women with POI, but also quantitatively evaluate changes in left ventricular function.

This study used TDI to assess the changes in left ventricular diastolic function in patients with POI. In this study, the E, E/A, and lateral e' in POI group were lower those in the controls group $(P<0.05$ or $P$ $<0.01)$, but no differences in septal e', average E/e', LAVI were found between the two groups $(P>0.05)$, but according to guidelines proposed by the ASE/EACI in 2016 for left ventricular diastolic function, ${ }^{4}$ the left ventricular diastolic function did not decrease in both two groups in this study. And the above suggested although there was no significant decrease in left ventricular diastolic function in patients with POI, there were still some signs of decreased diastolic function.

The reasons for the decline of left ventricular diastolic function due to $\mathrm{E}_{2}$ deficiency are more complicated, the main possible reasons are: an animal experiment showed that MCP-1 and MCP-5 mRNAs in the cardiomyocytes of ovariectomized rats, as the isoforms of chymase forming angiotensin II (Ang II), participate in the loss of normal heart function and structure caused by ovariectomy in rodents, including decreased left ventricular diastolic function (increased E/e') and increased LVEDD ${ }^{16}$ And one other animal experiment showed that $\mathrm{E}_{2}$ could improve the antioxidant capacity of cardiomyocytes in ovariectomized rats and play a protective effect on myocardium, ${ }^{17}$ then suggesting that lack of $E_{2}$ could lead to the reduction of myocardial antioxidant capacity, aggravation of fibrosis, and further reduction of left ventricular diastolic function; In addition, it had been reported that $\mathrm{E}_{2}$ deficiency would lead to reduced mitochondrial function of cardiomyocytes and inhibit NOs signaling, thereby inhibiting the GPR30 pathway, which together leaded to a decrease in left ventricular diastolic function. ${ }^{14}$

Changes in left ventricular diastolic function are often inseparable from changes in systolic function. However, there was no obvious abnormality in left ventricular systolic function when the left ventricular diastolic function in the POI group in conventional echocardiography was abnormal (there was no significant difference in LVEF between the two groups), suggesting that the use of conventional LVEF may not reflect the subclinical changes of left ventricular systolic function early.

This study used layer-specific myocardial strain and found that GLSendo, GLSmid, GLSepi, GCSendo, GCSmid, and GCSepi in the POI group were lower than those in the controls group, suggesting that there were reduced left ventricular systolic function in POI group, and layer-specific myocardial strain could more sensitively prompt the left ventricular systolic function impairment in POI patients than conventional twodimensional LVEF. The possible mechanisms for the decrease in layer-specific strains of the left ventricle in the POI group were: an animal experiment had shown that $\mathrm{E}_{2}$ enhanced cardiac systolic function by regulating the expression of cAMP-L-type $\mathrm{Ca}_{2}{ }^{+}$channel (cAMP-LTCC) related genes, and confered cardioprotection of against stress through the GPR30 non-genomic acute signaling pathway, ${ }_{18}{ }_{\text {so }}$ so lack of $\mathrm{E}_{2}$ might lead to decreased myocardial systolic function, appearing the result of this study; In addition, Cheng and other researches believed that there were estrogen receptors (ER) in the arterial wall and myocardial tissue, including $\mathrm{ER} \alpha, \mathrm{ER} \beta$, and G protein-coupled estrogen receptor (GPER), $\mathrm{E}_{2}$ could directly act on cardiomyocytes, enhance myocardial systolic function and reduce heart rate variability, ${ }^{19-21}$ then lack of $\mathrm{E}_{2}$ may directly lead to increased myocardial oxygen consumption and energy requirements, and decrease myocardial systolic function.

There is a certain gradient in the deformability of the three-layer myocardial structure of the normal left ventricle, the deformation of the myocardium from endocardium to epicardium is gradually decreasing. ${ }^{22}$ This study also confirmed this decreasing trend from endocardium to epicardium, both in the POI group 
and the controls group.

Through the ROC curve to analyze the efficacy of the layer-specific strain parameters in diagnosing the left ventricular systolic function in POI patients, we found that compared with other parameters, the AUC of GLSepi was largest $(\mathrm{AUC}=0.706, \mathrm{P}=0.000)$, indicating that it might have a higher diagnostic value for the detecting of left ventricular myocardial systolic function impairment in patients with POI.

But this study showed while using $-20.15 \%$ as the cut-off value of GLSepi, the sensitivity was $79.9 \%$, and the specificity was $54.7 \%$, the specificity was still low, the further research was needed in the later period.

\subsection{Study limitations}

Limitations of this study: (a) Since the hospital serum sex hormone testing equipment had been set 37pmol/l as the bottom line of estrogen, it was impossible to collect the E2 of all patients in the POI group; E2 and FSH levels of the normal group were not collected; (b) The study was still a preliminary study, and the sample size can be increased to further verify the results; (c) The follow-up study during HRT had not been conducted.

\section{CONCLUSIONS}

In summary, POI patients with normal LVEF may have subclinical left ventricular myocardial systolic impairment. The layer-specific myocardial strain can more sensitively detect the subclinical left ventricular systolic impairment in POI patients, and can be used as a new non-invasive method for early evaluation of the left ventricular systolic function impairment in POI patients.

\section{AUTHOR CONTRIBITIONS}

Li-xue Yin was responsible for study conceptualization, design, and supervision, Yu-lin Wang was responsible for performing echocardiography, data analysis, and writing manuscript, Mei Li was responsible for providing cases. All authors have revised and approved the final submission of the manuscript.

\section{REFERENCES}

1.European Society for Human Reproduction and Embryology (ESHRE) Guideline Group on POI, Webber L, Davies M, et al. ESHRE Guideline: management of women with premature ovarian insufficiency. Hum Reprod. 2016;31(5):926-937.

2.Roeters van Lennep JE, Heida KY, Bots ML, Hoek A; collaborators of the Dutch Multidisciplinary Guideline Development Group on Cardiovascular Risk Management after Reproductive Disorders. Cardiovascular disease risk in women with premature ovarian insufficiency: A systematic review and meta-analysis. Eur J Prev Cardiol. 2016;23(2):178-186.

3.Lang RM, Badano LP, Mor-Avi V, et al. Recommendations for cardiac chamber quantification by echocardiography in adults: an update from the American Society of Echocardiography and the European Association of Cardiovascular Imaging. J Am Soc Echocardiogr. 2015;28(1):1-39.e14.

4.Nagueh SF, Smiseth OA, Appleton CP, et al. Recommendations for the evaluation of left ventricular diastolic function by echocardiography: an update from the American Society of Echocardiography and the European Association of Cardiovascular Imaging. J Am Soc Echocardiogr. 2016;29(4):277-314.

5.Welt CK. Primary ovarian insufficiency: a more accurate term for premature ovarian failure. Clin Endocrinol (Oxf). 2008;68(4):499-509.

6.Laml T, Schulz-Lobmeyr I, Obruca A, et al. Premature ovarian failure: etiology and prospects. Gynecol Endocrinol. 2000;14(4):292-302.

7.Tsiligiannis S, Panay N, Stevenson JC. Premature Ovarian Insufficiency and Long-Term Health Consequences. Curr Vasc Pharmacol. 2019;17(6):604-609. 
8.Ebong IA, Watson KE, Goff DC Jr, et al. Age at menopause and incident heart failure: the Multi-Ethnic Study of Atherosclerosis. Menopause. 2014;21(6):585-591.

9.Esposito R, Santoro C, Sorrentino R, et al. Layer-specific longitudinal strain in Anderson-Fabry disease at diagnosis: A speckle tracking echocardiography analysis. Echocardiography. 2019;36(7):1273-1281.

10.Shi J, Pan C, Kong D, Cheng L, Shu X. Left Ventricular Longitudinal and Circumferential Layer-Specific Myocardial Strains and Their Determinants in Healthy Subjects. Echocardiography. 2016;33(4):510-518.

11.Shiino K, Yamada A, Scalia GM, et al. Early Changes of Myocardial Function After Transcatheter Aortic Valve Implantation Using Multilayer Strain Speckle Tracking Echocardiography. Am J Cardiol. 2019;123(6):956-960.

12.Skaarup KG, Iversen A, Jørgensen PG, et al. Association between layer-specific global longitudinal strain and adverse outcomes following acute coronary syndrome. Eur Heart J Cardiovasc Imaging. 2018;19(12):13341342 .

13.Haynes MP, Sinha D, Russell KS, et al. Membrane estrogen receptor engagement activates endothelial nitric oxide synthase via the PI3-kinase-Akt pathway in human endothelial cells. Circ Res. 2000;87(8):677682 .

14.Li S, Gupte AA. The Role of Estrogen in Cardiac Metabolism and Diastolic Function. Methodist Debakey Cardiovasc J. 2017;13(1):4-8.

15.Ribeiro RF Jr, Pavan BM, Potratz FF, et al. Myocardial contractile dysfunction induced by ovariectomy requires AT1 receptor activation in female rats. Cell Physiol Biochem. 2012;30(1):1-12.

16.Wang H, Sun X, Ahmad S, Su J, Ferrario CM, Groban L. Estrogen modulates the differential expression of cardiac myocyte chymase isoforms and diastolic function. Mol Cell Biochem. 2019;456(1-2):85-93.

17.Wu Q, Zhao Z, Sun H, at al. The cardioprotection of estrogen on solated global myocardial ischemia/reperfusion injury in ovariectomized rats. Zhongguo Ying Yong Sheng Li Xue Za Zhi. 2009;25(3):355360.(in Chinese)

18.Machuki JO, Zhang HY, Geng J, et al. Estrogen regulation of cardiac cAMP-L-type Ca2 ${ }^{+}$channel pathway modulates sex differences in basal contraction and responses to $\beta 2 \mathrm{AR}$-mediated stress in left ventricular apical myocytes. Cell Commun Signal. 2019;17(1):34. Published 2019 Apr 15.

19.Gorodeski GI. Update on cardiovascular disease in post-menopausal women. Best Pract Res Clin Obstet Gynaecol. 2002;16(3):329-355.

20.Losordo DW, Kearney M, Kim EA, et al. Variable expression of the estrogen receptor in normal and atherosclerotic coronary arteries of premenopausal women. Circulation. 1994;89(4):1501-1510.

21.Cheng SB, Graeber CT, Quinn JA, Filardo EJ. Retrograde transport of the transmembrane estrogen receptor, G-protein-coupled-receptor-30 (GPR30/GPER) from the plasma membrane towards the nucleus. Steroids. 2011;76(9):892-896.

22.Huttin O, Girerd N, Coiro S, et al. Association Between Layer-Specific Longitudinal Strain and Risk Factors of Heart Failure and Dyspnea: A Population-Based Study. J Am Soc Echocardiogr. 2019;32(7):854865.e8.

\section{Hosted file}

TABLES.pdf available at https://authorea.com/users/373759/articles/491360-assessment-ofleft-ventricular-myocardial-systolic-dysfunction-in-premature-ovarian-insufficiencyusing-echocardiographic-layer-specific-myocardial-strain-imaging

\section{Hosted file}


FIGURE 1.eps available at https://authorea.com/users/373759/articles/491360-assessment-ofleft-ventricular-myocardial-systolic-dysfunction-in-premature-ovarian-insufficiencyusing-echocardiographic-layer-specific-myocardial-strain-imaging

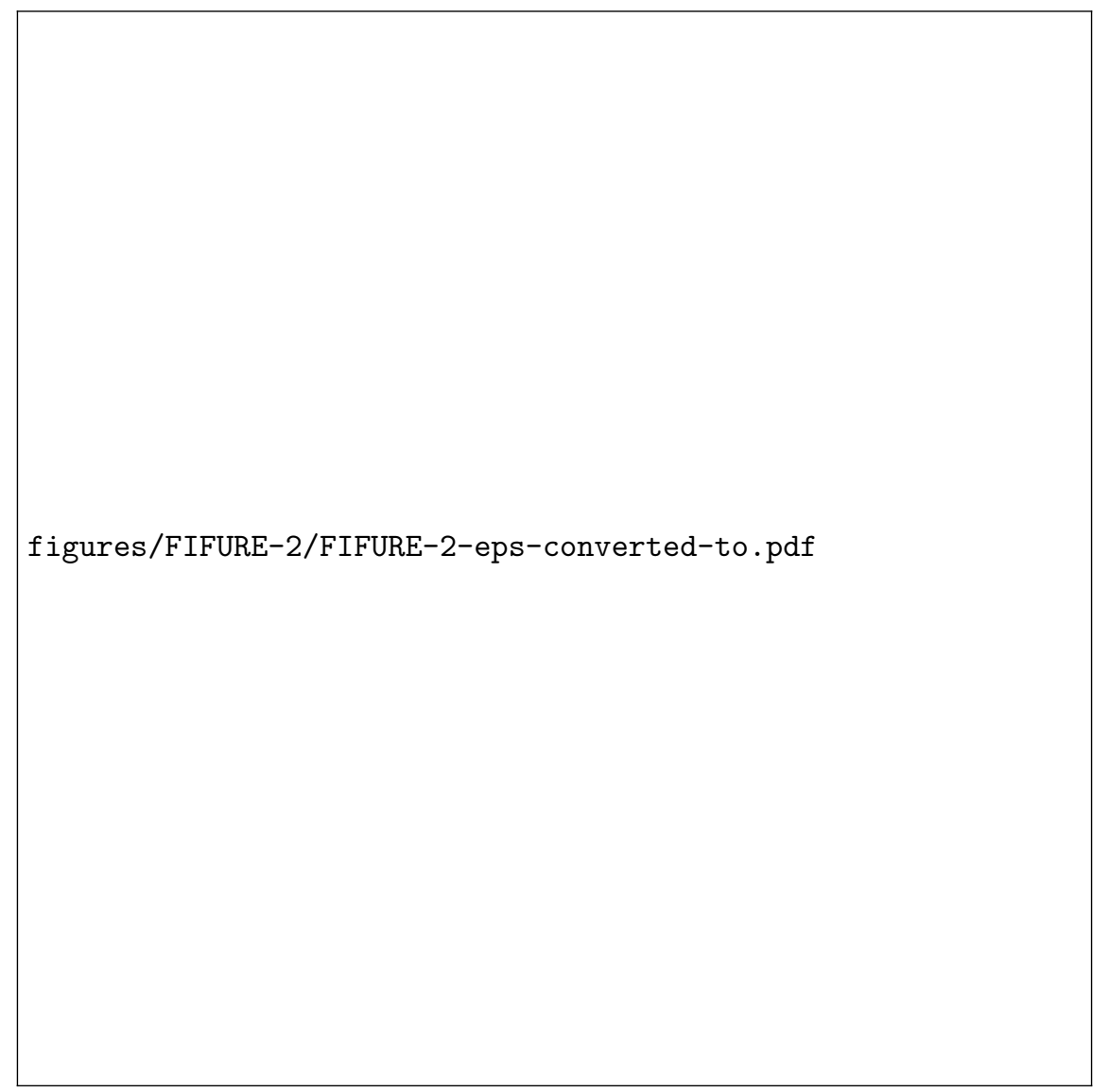

\section{Hosted file}

FIGURE 3.eps available at https://authorea.com/users/373759/articles/491360-assessment-ofleft-ventricular-myocardial-systolic-dysfunction-in-premature-ovarian-insufficiencyusing-echocardiographic-layer-specific-myocardial-strain-imaging

\section{Hosted file}

FIGURE 4.eps available at https://authorea.com/users/373759/articles/491360-assessment-ofleft-ventricular-myocardial-systolic-dysfunction-in-premature-ovarian-insufficiencyusing-echocardiographic-layer-specific-myocardial-strain-imaging 